\title{
Prácticas ambientales y de riesgos laborales: Caso de estudio en una empresa del sector calzado en Bucaramanga, Santander
}

Environmental and occupational risk practices: A case study of a company in the footwear sector in Bucaramanga, Santander

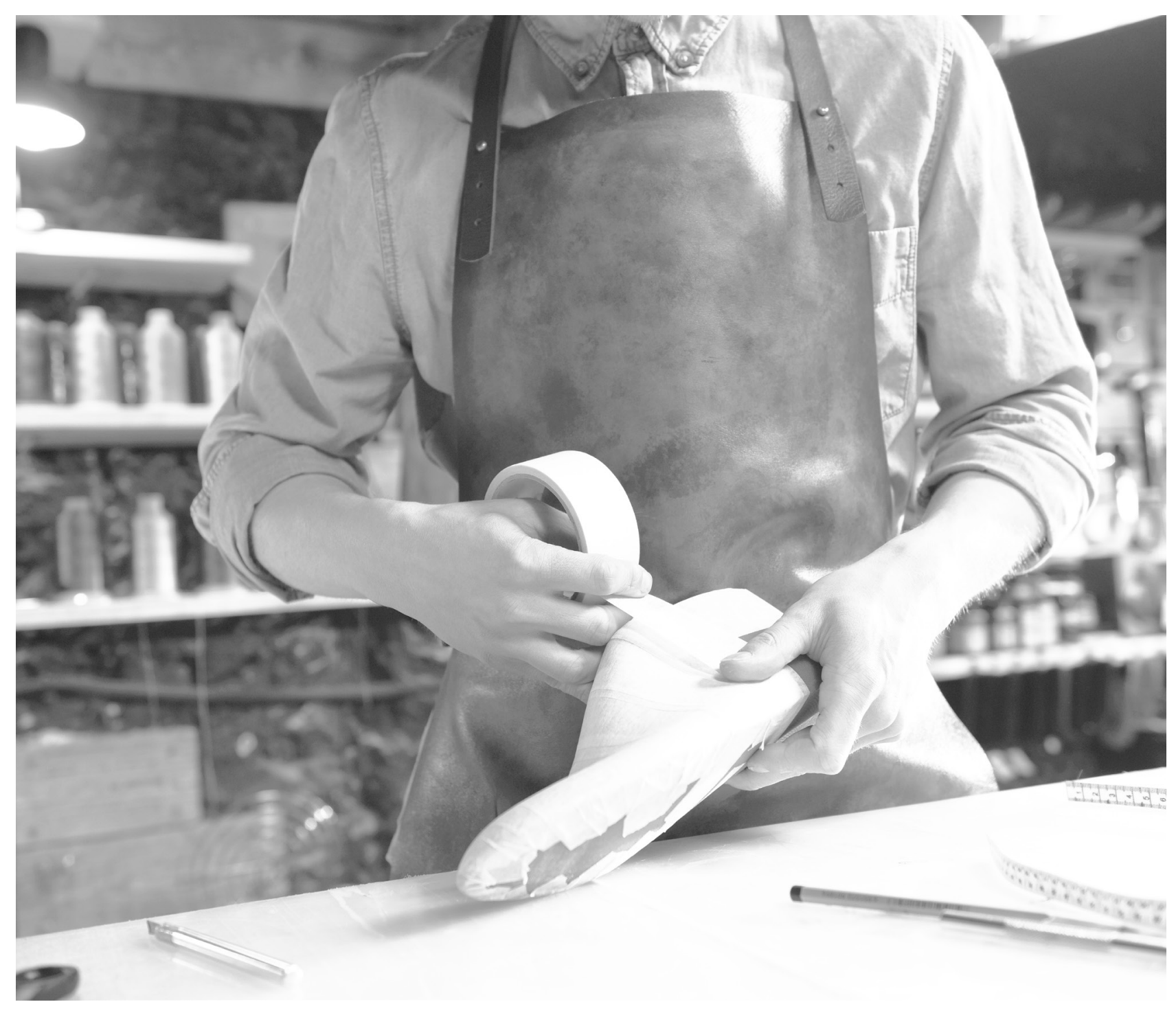




\title{
Prácticas ambientales y de riesgos laborales: Caso de estudio en una empresa del sector calzado en Bucaramanga, Santander ${ }^{1}$ Environmental and occupational risk practices: A case study of a company in the footwear sector in Bucaramanga, Santander
}

\author{
Cindy Tatiana Daza Ríos ${ }^{2}$, Irma Soto Vallejo ${ }^{3}$, Alejandra Garay Salamanca ${ }^{4}$
}

Artículo recibido en febrero 24 de 2020; artículo aceptado en mayo 19 de 2020

Este artículo puede compartirse bajo la Licencia Creative Commons Atribución-NoComercial-Compartirlgual 4.0 Internacional y se referencia usando el siguiente formato: Daza, C. T., Soto, I. y Garay, A. (2020). Prácticas ambientales y de riesgos laborales: Caso de estudio en una empresa del sector calzado en Bucaramanga, Santander. I+D Revista de Investigaciones, 15 (2), 98-106. DOI: https://doi.org/10.33304/revinv.v15n2-2020009

\begin{abstract}
Resumen
Las prácticas ambientales y riesgos laborales gestionan la sostenibilidad y permiten que las empresas funcionen considerando aspectos ambientales, sociales y económicos, lo que las hace más competitivas. A partir de esta premisa, la presente investigación se propone reconocer las prácticas ambientales y de seguridad y salud en el trabajo en una microempresa fabricante de calzado en Bucaramanga, Colombia, así como estudiar la relación entre dichas prácticas en la empresa de estudio. Para esto, se utilizó como diseño metodológico el estudio de caso, con enfoque mixto, corte transversal y alcance exploratorio descriptivo, utilizando instrumentos como la encuesta, la entrevista semiestructurada y la observación. La validez interna del estudio se aseguró por medio de triangulación de resultados por fuentes. Los resultados muestran cómo las prácticas ambientales y de riesgos laborales objeto de investigación en la empresa caso de estudio son incipientes o están ausentes, debido a la falta de políticas institucionales para su promoción. También se encontró que es evidente una articulación entre dichas prácticas ambientales y laborales.
\end{abstract}

Palabras clave: Desarrollo sostenible, prácticas ambientales, riesgos laborales, seguridad laboral.

\footnotetext{
${ }^{1}$ Artículo estudio de caso, de enfoque mixto, de corte transversal, resultado de un trabajo de investigación culminado de la Maestría en Desarrollo Sostenible y Medio Ambiente, perteneciente al área de Desarrollo Sostenible, subárea de Prácticas Laborales y Ambientales, desarrollado en el grupo de investigación SINERGIA, de la Universidad de Investigación y Desarrollo (UDI), financiado con recursos de la investigadora principal. Dirección: calle 9 n. $23-55$ (Bucaramanga, Colombia). PBX: (057) 6352525. Fecha de inicio: 2017. Fecha de terminación: 2018.

${ }^{2}$ Bacterióloga y laborista clínica de la Universidad Industrial de Santander, especialista en Salud Ocupacional de la Universidad Manuela Beltrán y magíster en Desarrollo Sostenible y Medio Ambiente de la Universidad de Manizales. Docente investigadora del grupo de investigación SINERGIA, de la Universidad de Investigación y Desarrollo (UDI) (Bucaramanga, Colombia). Dirección: calle 9 n. 23-55 PBX: (057) 6352525. ORCID ID: https:// orcid.org/0000-0003-2749-3602. Correo electrónico institucional: cdaza1@udi.edu.co.

${ }^{3}$ Magíster en Ciencias Económicas de la Universidad Nacional de Colombia Sede Medellín. Profesora titular, investigadora y directora del Centro de Investigaciones en Medio Ambiente y Desarrollo (CIMAD), Universidad de Manizales (Colombia). Dirección: carrera 9 n. ${ }^{\circ}$ 19-03. PBX: (6) 8879680, exts. 1370-1377. ORCID ID: https://orcid.org/0000-0003-3871-4853. Correo electrónico institucional: irma@umanizales.edu.co.

${ }^{4}$ Magíster en Salud Ocupacional de la Universidad del Valle. Profesora de proyecto de grado en la Maestría en Desarrollo Sostenible y Medio Ambiente de la Universidad de Manizales. Dirección: carrera 9 n. 19-03. PBX: (6) 8879680, exts. 1370, 1377. ORCID ID: https://orcid.org/0000-00032488-6857. Correo electrónico institucional: f.t.alejagaray@umanizales.edu.co.
} 


\begin{abstract}
Environmental practices and occupational risks manage sustainability and allow companies to operate considering environmental, social, and economic aspects, which makes them more competitive. Based on this premise, this research aims to recognize environmental, and health and security practices at work in a shoe manufacturing microenterprise in Bucaramanga, Colombia, as well as to study the relationship between these practices in the company studied. For this, the case study was used as a methodological design, with a mixed approach, cross-sectional and descriptive exploratory scope, using instruments such as surveys, semi-structured interviews, and observation; the internal validity of the study was ensured by triangulation of results by source. The results show how the environmental and occupational risk practices object of investigation in the case study company are incipient or absent, due to the lack of institutional policies for its promotion. It was also found that articulation between these environmental and labor practices are evident.
\end{abstract}

Keywords: Sustainable development, environmental practices, occupational risks, occupational security.

\section{Introducción}

De acuerdo con los postulados de Volpentesta (2016), las empresas han aumentado la incorporación de prácticas de Responsabilidad Social Empresarial (RSE). Desde los años 80 se ha estado emitiendo normatividad en el ámbito internacional que busca orientar a las organizaciones para que puedan desarrollar sus actividades de forma sostenible. En Colombia, desde los años 90 se han emitido las bases legales para poder mostrar a las empresas cómo implementar estas prácticas, según lo ha definido el Congreso de la República de Colombia (1993). Pero, a pesar de las guías y lineamientos internacionales y nacionales difundidos desde hace varios años, no todas las empresas del país han adoptado las medidas necesarias, como es el caso de la microempresa objeto de estudio. Esta situación ha ocasionado problemas sociales y ambientales como contaminación, enfermedad y accidentalidad laboral.

La mayoría de personas trabaja en las organizaciones ejecutando diferentes actividades laborales, con el objetivo de desarrollar sus competencias personales y profesionales, y reciben a cambio dinero para poder cubrir sus necesidades. Así mismo, las empresas elaboran productos o bienes para satisfacer las necesidades de la sociedad, con lo que contribuyen al crecimiento económico del país. Por tanto, la empresa es fundamental para el desarrollo de la sociedad, de acuerdo con lo establecido en el artículo 333 de la Constitución Política de Colombia de 1991. Sin embargo, en la ejecución de estas actividades laborales se pueden generar impactos negativos que afectan al medioambiente y la salud de los trabajadores. Por esto, es necesario educar y sensibilizar a los empleadores y trabajadores de todos los sectores económicos, con el objetivo de promover el desarrollo de las actividades productivas haciendo uso de prácticas sostenibles; es decir, que los sectores busquen mitigar y controlar los impactos ambientales negativos y promover los positivos, así como fomentar la ejecución de actos seguros y hábitos saludables, de forma que se contribuya al aspecto económico, social y ambiental, lo que, a su vez, se vería reflejado en un mejoramiento de la calidad de vida de los empleados.

Las micro, pequeñas y medianas empresas (mipymes) constituyen la mayoría de las empresas existentes en Latinoamérica y el mundo. Estas presentan características propias, como informalidad y ausencia de prácticas ambientales, de seguridad y salud en el trabajo, según Ortiz et al. (2013). De acuerdo con la Asociación Colombiana de Industriales de Calzado, el cuero y sus manufacturas (Acicam), en Colombia, en el sector de cuero, calzado y marroquinería, existen alrededor de 13.000 empresas en el eslabón de la transformación, y 15.000 , en el sector de comercialización. Esta actividad se realiza en 28 de los 32 departamentos del país; en Santander se ubica el $33 \%$ de las empresas de manufactura (Acicam y Fedecuero, 2013).

Debido al alto número de empresas de este sector en el país y la región, esta actividad es de alta importancia económica en la región, tal como lo comunican Acicam y Fedecuero (2013). La predominancia de mipymes es común en este sector, y debido a las particularidades de estas, es importante ejecutar estudios para conocer las condiciones de salud y de trabajo del recurso humano, así como las características del proceso productivo que realizan, en el cual se emplean diferentes sustancias químicas aromáticas, como el benceno y tolueno, presentes en adhesivos, solventes y colorantes, que pueden generar contaminación. Por tanto, el objetivo de esta investigación es describir las prácticas ambientales y de riesgos laborales en el trabajo en una mipyme fabricante de calzado y la relación entre dichas prácticas.

El presente artículo se estructura de la siguiente manera: inicia con la revisión teórica del tema de investigación, seguido de la metodología utilizada, y finaliza con los resultados y conclusiones del caso de estudio analizado. 


\section{Riesgos laborales y ambientales en el proceso de fabricación de calzado}

La fabricación de calzado es uno de los trabajos más antiguos. En este proceso se utilizan químicos que contienen benceno y tolueno, que, de acuerdo con un estudio realizado por Azari et al. (2012), en el que monitorearon durante 3 meses consecutivos la exposición al benceno y tolueno, se encontró que la severidad de la exposición de los zapateros al tolueno se correlacionó significativamente con la cantidad de zapatos fabricados y la cantidad de pegamento utilizado en el proceso. También indica que los trabajadores, en el desarrollo de sus actividades laborales, están expuestos a estos químicos en unos niveles de concentración mayores a los límites establecidos, lo que se traduce en un riesgo de presentar cáncer u otra patología laboral asociada a esta exposición a benceno y tolueno. Por esto, es necesario gestionar las condiciones adecuadas, para garantizar un ambiente de trabajo sano, una mejor calidad de vida y evitar la ocurrencia de enfermedades laborales (Azari et al., 2012).

Otra investigación realizada por Emanuelli et al. (2016) muestra cómo los trabajadores del estudio que hacían parte del proceso de fabricación de calzado tenían un riesgo significativo de adquirir cáncer sinusal. Por su parte, Flores-Hernández et al. (2012) ahondaron en el daño toxicológico de empleados con exposición crónica a solventes orgánicos en la industria de cuero y calzado, a través de la manifestación de síntomas psicológicos. Como resultado se encontró que el 9,2 \% de los empleados presentó daño neurológico -principalmente mujeres trabajadoras en empresas de calzado-. El daño neurológico se manifestó por labilidad, fatiga y disminución de la sociabilidad (Flores-Hernández et al., 2012).

Estos estudios sobre neurotóxicos laborales son de interés en diferentes países en los que se han realizado estudios, como, por ejemplo, en India y Venezuela, así como en México y España, donde se han comparado los contextos de estos países, y donde se indica que los efectos tóxicos de las sustancias químicas en ambientes laborales son una actividad compleja que requiere un trabajo coordinado y de colaboración preventiva para generar un conocimiento más amplio de las sustancias causales y sus efectos (Vicente-Herrero et al., 2016). Adicionalmente, en un estudio realizado sobre trabajadores palestinos se menciona que:

La exposición a largo plazo a disolventes orgánicos se asocia con irritación neuropsiquiátrica y de la membrana mucosa. En los países en desarrollo, los esfuerzos para asegurar un buen ambiente de trabajo son inadecuados y la protección contra la exposición a químicos a menudo se descuida. (Nijem et al., 2001)
De acuerdo con dicha investigación, los trabajadores reportaron una alta prevalencia de problemas neuropsiquiátricos y de membranas mucosas como dolor de cabeza, irritabilidad mental, hormigueo en las extremidades y dolor en los ojos, lo que es resultado de la exposición a solventes orgánicos volátiles, según plantean Nijem et al. (2001).

Por otra parte, en el aspecto ambiental se han realizado investigaciones sobre las características reológicas de adhesivos base agua y base disolvente, fluidos de interés para la industria del calzado, y se ha asegurado que el uso de adhesivos base agua reduciría los impactos negativos en la salud y el medioambiente (González et al., 2016). Esto es coherente con un estudio realizado por Gangopadhyay et al. (2011) en la India, en el cual se indica que uno de los riesgos más importantes a los que se exponen los trabajadores del sector calzado es al químico de adhesivos y pegamentos industriales, causantes de diversas enfermedades respiratorias, neurotoxicidad y cáncer. Gangopadhyay et al. (2011) observaron que las partículas de polvo y los adhesivos afectan la capacidad pulmonar de los trabajadores de calzado. Además, esto coincide con lo reportado por Rodríguez et al. (2014), que investigaron la asociación de niveles de ácido hipúrico en orina y los efectos neurológicos de trabajadores de empresas de calzado. Este estudio encontró que los trabajadores expuestos presentaron concentraciones mayores que un grupo no expuesto; además, encontró asociación con signos y síntomas como cansancio, irritabilidad, cambio de humor y adormecimiento de manos y pies. El $60 \%$ refirió no usar elementos de protección personal; el $30 \%$, mascarillas antipolvo, y el $10 \%$, respiradores antivapores orgánicos, lo que sugirió que las alteraciones del sistema nervioso pudieran estar asociadas a la exposición a Tolueno, ausencia de ventilación y poco uso de protección personal (Rodríguez et al., 2014).

Las investigaciones anteriores dan cuenta de la necesidad de gestionar de mejor forma la salud ocupacional y prácticas ambientales en el sector calzado. Actualmente existe una tendencia hacia la sostenibilidad, y esta impulsa la salud ocupacional (Meyer, 2013). Por tanto, es necesario generar más conocimiento en estos temas. Las mipymes colombianas enfrentan dificultades en cuanto a competitividad, para lo que se han desarrollado investigaciones que buscan contribuir al mejoramiento de esta (Montoya et al., 2010). Se deben considerar estrategias para mejorar la sostenibilidad y competitividad de la empresa gestionando adecuadamente la salud ocupacional y la gestión ambiental (González, 2011; Rodríguez y van Hoof, 2010). Por ejemplo, en la literatura española se ha evidenciado cómo han incrementado en los últimos años las investigaciones sobre gestión ambiental en las empresas (Rubio et al., 2007). También se ha evidenciado 
que para considerar a la dirección de las empresas enfocadas en la protección ambiental como una buena estrategia, se deben involucrar las partes interesadas (Plaza et al., 2011).

\section{Metodología}

\section{Tipo de estudio}

La investigación que se desarrolló tuvo un alcance exploratorio-descriptivo, con enfoque mixto, y consideró los enfoques cualitativo y cuantitativo, un diseño de caso de estudio y de corte transversal.

\section{Participantes}

La empresa utilizada para el caso de estudio es una fábrica de calzado de la ciudad de Bucaramanga, dedicada a la elaboración de calzado en cuero para damas y caballeros, de tipo casual y formal; es decir, calzado deportivo y clásico formal. La empresa es el resultado de un proyecto que inició en una universidad de Santander, que recibió financiación externa para su creación y constitución legal.

\section{Materiales e instrumentos}

La muestra se seleccionó de forma no probabilística y considerando la saturación teórica. La microempresa caso de estudio corresponde a una fábrica de calzado con menos de 10 trabajadores, de pequeño tamaño, con baja presencia de tecnología, trabajo artesanal y poca o ninguna capacitación de los gerentes en temáticas de sistemas o prácticas de gestión ambiental, de seguridad y salud laboral. Los anteriores criterios se verificaron en la entrevista con el gerente, en la que se presentó el propósito de la investigación y se solicitó el aval para el desarrollo de esta.

- Unidad de análisis: Empresa caso de estudio.

- Técnicas e instrumento: Entrevista semiestructurada sobre prácticas ambientales, de seguridad y salud laboral, validada por dos expertos, uno metodológico, magíster en Epidemiología y docente con amplia experiencia en investigación y experiencia especifica en estas áreas disciplinares (investigador júnior de Minciencias); el otro con amplia experiencia en el área disciplinar de esta investigación. Cuestionario sobre condiciones de salud y trabajo elaborado por el Ministerio del trabajo de Colombia. Finalmente, la observación con base en la (GTC-45) para la identificación de peligros y evaluación de riesgos por medio de un diario de campo.

La empresa fue seleccionada por saturación teórica, buscando proponer teorías e inferir conclusiones representativas para el sector.
- Triangulación de resultados: Se realizó por medio de fuente de datos.

\section{Principios éticos}

Teniendo en cuenta las consideraciones éticas, antes de proceder con la recolección de información, cada participante en la investigación leyó el consentimiento informado y aceptó la participación en la investigación de forma voluntaria. En el consentimiento informado se garantiza la confidencialidad de la información recolectada, se asegura que los datos solo serán conocidos por los investigadores y se comunican los derechos de los participantes en el estudio.

\section{Resultados}

Inicialmente, se presentarán los resultados encontrados en la investigación y, posteriormente, se realizará la discusión.

La fábrica de calzado utilizada como caso de estudio está formal y legalmente constituida desde el año 2015. Esta microempresa es el resultado de un proyecto que inició en una universidad de Santander. Durante sus primeros meses de funcionamiento, la inyección de capital provino de sus propietarios. En el año 2015 la fábrica ganó una convocatoria de emprendimiento para financiar su operación de acuerdo con la legislación vigente. La financiación recibida exigía, entre otros, la implementación del SG-SST. Por tanto, la empresa desarrolló actividades asociadas a seguridad y salud en el trabajo. Es de resaltar que la característica diferenciadora de esta fábrica consiste en la elaboración de calzado personalizado.

Por otra parte, como características sociodemográficas importantes de esta fábrica, se resalta que el gerente tiene formación universitaria, al igual que todos los trabajadores de esta empresa; es decir, el $100 \%$ del recurso humano es altamente calificado. Las edades de los trabajadores oscilan entre 26 y 35 años, por lo que se considera una población joven, y el $100 \%$ de los empleados son hombres.

En cuanto a las condiciones de salud y seguridad en el trabajo, se resalta que los cargos establecidos en la empresa son gerente y diseñador, corte, soladura y montado. El $100 \%$ del personal manifestó trabajar en una jornada de 8 horas, esto es, lo establecido por la legislación. La totalidad de los empleados realiza pausas activas cada vez que las quiera realizar, y todos se encuentran afiliados a seguridad social. Además, el $100 \%$ cuenta con un sueldo fijo, y los niveles de estrés reportados en una escala de 1 a 10 , donde 10 es el máximo nivel, se ubican en una escala de 4 a 7 , de los cuales los operarios reportaron niveles de 4 y 5 , y el gerente reportó un nivel de 7 . 
En cuanto a condiciones sanitarias, el $100 \%$ de los empleados considera que son buenas, ya que se cuenta con agua potable, hay un inodoro, que es suficiente para el número de trabajadores, y está en buen estado, aunque no existen armarios para guardar las cosas en el lugar de trabajo ni vestidores.

Los principales peligros identificados y señalados en el lugar de trabajo corresponden a peligros físicos, como temperatura, iluminación y ruido. También se encontraron peligros biomecánicos por malas posturas y esfuerzos realizados en las actividades laborales en el cargo de cortador. Adicionalmente, el $100 \%$ de los trabajadores se siente satisfecho con su actividad y considera que puede aplicar sus conocimientos y habilidades. No se han presentado eventos laborales. Todos manifiestan estar en buen estado de salud y consideran el lugar de trabajo como seguro.

De acuerdo con la información obtenida, se pueden resaltar como aspectos para mejorar la implementación de prácticas de seguridad y salud en el trabajo realizar exámenes médicos ocupacionales, dado que se están omitiendo, y la legislación establece que, por ley, se deben realizar al momento de ingreso, periódicos (anualmente) y al momento de egreso del sitio de trabajo. También es importante que se implementen los sistemas de vigilancia epidemiológica, teniendo en cuenta la identificación, evaluación y priorización de peligros, dado que se evidencia el no desarrollo de estas. A su vez, según la actividad económica que se realiza, se debe implementar un sistema de vigilancia epidemiológica osteomuscular y uno químico. Así mismo, es necesario entregar elementos de protección personal (EPP) adecuados y en buen estado para los cargos que aplican; por ejemplo, protección respiratoria, como mascarilla con filtro para vapores orgánicos, para el solador; tapabocas y guantes, para el cortador, así como zapatos seguros y cómodos para todo el personal y jean gramaje 14 para los operarios.

Como factores de riesgo que podrían ocasionar accidentalidad, se identifica la necesidad de cumplir las metas de producción, entrega de EPP de mala calidad e inadecuadas condiciones de orden y aseo. Los principales riesgos identificados por el personal debido a su trabajo fueron quemaduras por químicos o vapores. Por último, los empleados reportaron un $0 \%$ de actividades recreativas o de integración organizadas por la empresa.

Como resultado de la observación realizada, se puede determinar que la fábrica cuenta con la señalización y los elementos necesarios para atención a emergencias, como extintor, camilla y botiquín. Además, se observó la documentación del sistema de gestión acorde a las actividades desarrolladas por la empresa, pero no se evidencia cumplimiento de la implementación, como diligenciamiento de formatos de inspecciones, reuniones de vigía de SST, reuniones de comité de convivencia laboral (CCL), entrega de matriz de EPP, entre otros. No se observa el uso de EPP, ni la existencia de recipientes adecuados para la clasificación de residuos generados. Tampoco se cuenta con el contrato de una empresa especial para la disposición final de residuos peligrosos, por lo que los residuos tóxicos se disponen como residuos ordinarios, lo que ocasiona contaminación, por la gestión inadecuada.

En resumen, se observa que aunque el SG-SST está diseñado y documentado, no se implementa de forma correcta, y se debe trabajar bastante en este aspecto. Además, se hace evidente el conocimiento de los empleados sobre temáticas de SST y la implementación de prácticas que buscan la prevención de eventos laborales. Por otra parte, las únicas prácticas proambientales identificadas son la reutilización de cuero y el uso de algunos insumos amigables con el ambiente, pero no se cuenta con la infraestructura necesaria para reciclar y clasificar adecuadamente los residuos, y realizar una disposición final adecuada de los residuos peligrosos. La microempresa manifestó que la mejor forma para capacitar a los empleados en estas temáticas es con el ejemplo, capacitaciones con actividades y material práctico que utilice medios audiovisuales.

\section{Discusión de resultados}

\section{Triangulación por fuentes}

La triangulación por fuentes se realizó al recolectar información por medio de la entrevista semiestructurada sobre prácticas ambientales y de seguridad laboral a más de una fuente; es decir, a más de un trabajador de la microempresa caso de estudio. Las prácticas reportadas se pueden observar en las Tablas 1 y 2.

Tabla 1

Prácticas de seguridad y salud en el trabajo

Prácticas de seguridad y salud en el trabajo desarrolladas en la empresa caso de estudio

Diseño y documentación del Sistema de Gestión de Seguridad y Salud en el Trabajo

Sensibilización sobre la finalidad de la seguridad y salud en el trabajo

Capacitaciones a los trabajadores sobre primeros auxilios, atención de emergencias y manejo de químicos 


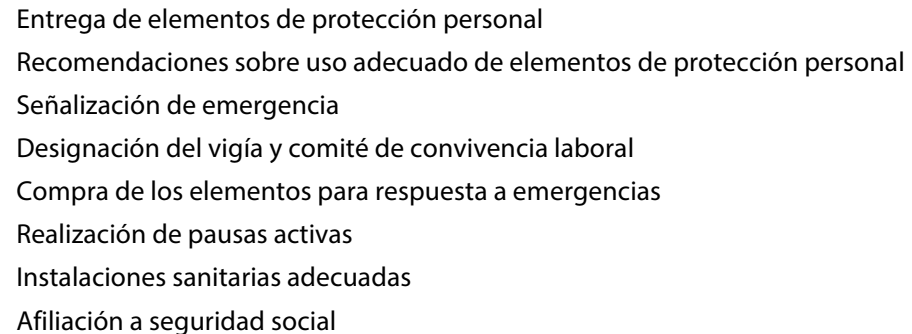

Fuente: Autores.

Tabla 2

Prácticas ambientales

Prácticas ambientales en la empresa caso de estudio

Reutilización de cuero en la elaboración de otros productos complementarios al calzado

Uso de insumos "ecoamigables" no tóxicos

Ahorro de agua (cerrando la llave mientras se cepilla los dientes, entre otras)

Apagado de luz a la hora del almuerzo y al salir del trabajo Utilización de cicla como medio de transporte

Fuente: Autores.

Al comparar los resultados obtenidos de la entrevista realizada a trabajadores con los resultados obtenidos por la observación realizada a la microempresa objeto de estudio, se encontró que estos coinciden en que las prácticas ambientales y laborales están en un estado incipiente o ausente. En concreto se evidencia que en la fábrica se realizan algunas prácticas de seguridad y salud en el trabajo, como reutilización de cuero y ahorro de agua y luz, lo que muestra la necesidad de trabajar de manera ardua y constante para cambiar y mejorar su situación.

Así mismo, se evidencia una relación entre las dos prácticas estudiadas, en lo referente al peligro químico, ya que, si se realiza un inadecuado manejo de las sustancias químicas, estas se pueden convertir en fuente de riesgo para la salud y seguridad de trabajadores, lo que puede generar cáncer, alergias, enfermedades neurológicas, incendios y explosiones, entre otros; así como para el medioambiente, porque son fuente de contaminación para el aire, agua y suelo. Esta misma situación se presenta para peligros de tipo físico y biológico; por tanto, deben gestionarse adecuadamente para cuidar el medioambiente y los trabajadores, a fin de eliminar fuentes de peligro comunes.

Los estudios en el ámbito internacional muestran cómo en los últimos años se está investigando sobre los impactos negativos generados por el funcionamiento de las empresas en el medioambiente y en la sociedad, además de cómo hacer para que estas, por medio de la responsabilidad social empresarial y el involucramiento de partes interesadas, tomen decisiones estratégicas y operen considerando aspectos ambientales y sociales, sin considerar los aspectos económicos como fin único para tomar decisiones. A pesar de esto, se requiere más investigación en esta área, pues los resultados obtenidos evidencian una subestimación del riesgo y comportamientos inseguros por parte del gerente y trabajadores de la empresa caso de estudio, así como muy pocos comportamientos proambientales. Esto muestra la necesidad de estudiar esta temática fundamental para la sostenibilidad de las empresas.

Los resultados obtenidos en esta investigación evidencian cómo durante el proceso de fabricación de calzado se utilizan químicos, como lo comprobó Azari et al. (2012); por consiguiente, se coincide con los autores en la necesidad de gestionar adecuadamente el medioambiente de trabajo para evitar la ocurrencia de enfermedades laborales. Es necesario, especialmente, diseñar e implementar medidas de prevención y control enfocadas en la prevención de enfermedades neuropsiquiátricas y/o cáncer, como lo reportaron Emanuelli et al. (2016) y Flores-Hernández et al. (2012). Finalmente, los resultados de esta investigación permiten resaltar la importancia de gestionar practicas sostenibles, tal como fue reportado por Meyer (2013). De esta forma se gestionará la competitividad y permanencia de la empresa caso de estudio.

\section{Conclusiones}

La fábrica de calzado objeto de estudio presenta ausencia o niveles incipientes de implementación de prácticas de seguridad, salud laboral y ambiental; por consiguiente, esta microempresa no está ejecutando prácticas sostenibles que contribuyan al desarrollo sostenible, lo que afecta su competitividad y el bienestar de la sociedad.

Se evidencia la existencia de una articulación en la ejecución de prácticas ambientales y de seguridad y salud en el trabajo en la microempresa caso de estudio; por tanto, las directrices que se tomen asociadas a estas deben considerarse de forma conjunta con el objetivo de optimizar recursos y efectividad, lo que busca cuidar el medioambiente y los trabajadores. 
Los trabajadores expuestos a químicos en la empresa fabricante de calzado tienen más riesgo de sufrir cáncer o enfermedades neuropsiquiátricas, como lo confirman los estudios de Emanuelli et al., (2016); Flores-Hernández et al. (2012); Vicente-Herrero et al. (2016); Nijem et al. (2001); Nije $\mathrm{m}$ et al. (2001) y Gangopadhyay et al. (2011). Sin embargo, los empleados de la empresa caso de estudio desconocen este riesgo, y en sus condiciones de trabajo predomina el no uso de elementos EPP, falta de capacitación y ausencia de un adecuado manejo y disposición final de estos residuos, factores que hacen que el riesgo aumente.

Los trabajadores de la fábrica de calzado, además de exponerse a peligros químicos, también se exponen a peligros de origen físico, debido a condiciones inadecuadas de iluminación, temperatura, ruido y peligros biomecánicos, y a posturas prolongadas y movimientos repetitivos. Estos peligros se deben tener en cuenta a la hora de implementar acciones para evitar la ocurrencia de eventos laborales.

\section{Recomendaciones}

Es necesario realizar más estudios que permitan conocer la situación de las mipymes fabricantes de calzado en la región y el país en relación con la ejecución de prácticas ambientales, de seguridad y salud en el trabajo o laborales. Lo anterior, a fin de generar información científica de calidad y utilidad, cuyos resultados permitan a todo el sector del calzado local y nacional mejorar su situación, de forma que estas empresas sean más competitivas y sostenibles.

La empresa estudio de caso debe mejorar el estado de ejecución de prácticas ambientales y de seguridad y salud en el trabajo. Para esto, se considera necesario sensibilizar a los trabajadores y gerentes, capacitarlos por medio de actividades prácticas y herramientas audiovisuales. Dichas actividades deben ser promovidas por la dirección, dado que el liderazgo es esencial y necesario para poder cumplir con este objetivo que beneficia a la empresa, los trabajadores y la comunidad en general. Estas deben ser políticas institucionales formales que involucren las partes interesadas para que sean efectivas.

\section{Agradecimientos}

Especial agradecimiento a los trabajadores y dueños de la empresa caso de estudio, y al Dr. Álvaro Javier Idrovo, por su orientación en el proceso investigativo.

\section{Referencias}

Asociación Colombiana de Industriales del Calzado, el Cuero y sus Manufacturas [Acicam], y [Fedecuero].
(2013). Plan de negocios del Sector de Cuero, Calzado y Marroquinería: Una respuesta para la transformación productiva. https://www.urosario.edu.co/urosario files/portalurosario/7e/7e3381ed-ab23-4350-a9718bbc6dc535bc.pdf.

Azari, M. R., Hosseini, V., Jafari, M. J., Soori, H., Asadi, P. y Mousavion, S. M. A. (2012). Evaluation of Occupational Exposure of Shoe Makers to Benzene and Toluene Compounds In Shoe Manufacturing Workshops in East Tehran. Tanaffos, 11(4), 43-49.

Congreso de la República de Colombia. (1993). Ley 99 de 1993. Por la cual se crea el Ministerio del Medio Ambiente.

Constitución Política de Colombia (1991). Título 12 - Del régimen económico y de la hacienda pública. Capítulo 1: De las disposiciones generales. Artículo 333. https:// www.constitucioncolombia.com/titulo-12/capitulo-1

Emanuelli, E., Alexandre, E., Cazzador, D., Comiati, V., Volo, T., Zanon, A., Scapellato, M. L., Carrieri, M., Martini, A. y Mastrangelo, G. (2016). A Case-Case Study on Sinonasal Cancer Prevention: Effect from Dust Reduction in Woodworking and Risk of Mastic/ Solvents in Shoemaking. Journal of Occupational Medicine and Toxicology, 11(1), 35. https://doi. org/10.1186/s12995-016-0124-7

Flores-Hernández, C., Páramo-Castillo, D., Huerta-Franco, M. R., Hernández, J., Cappacione, K., Vargas-Luna, M., Díaz de León-Morales L. V. y Morales-Mata, I. (2012). Evaluación psico-toxicológica de los trabajadores con exposición crónica a solventes orgánicos en las industrias del cuero y del calzado. Ciencia y Trabajo, (43), 129-134.

Gangopadhyay, S., Ara, T., Dev, S., Ghoshal, G. y Das, T. (2011). An Occupational Health Study of the Footwear Manufacturing Workers of Kolkata, India. Studies on Ethno-Medicine, 5(1), 11-15.

González, M. E., Campos, J. J., Valle, J. D. y Marmolejo, D. M. (2016). Caracterización de propiedades reológicas en fluidos de interés para la industrial del calzado: Búsqueda de mejorar la salud y el ambiente. Jóvenes en la Ciencia, 2(1), 1904-1908.

González, S. (2011). Sistemas integrados de gestión, un reto para las pequeñas y medianas empresas. Escenarios, 9(1), 69-89.

Meyer, F. E. (2013). Sustentabilidad: Un nuevo impulso a la salud ocupacional. Ciencia y Trabajo, 15(47), 63-66.

Montoya, A., Montoya, I.Y Castellanos, O. (2010). Situación de la competitividad de las pyme en Colombia: elementos actuales y retos. Agronomía Colombiana, 28(1), 107-117.

Nijem, K., Kristensen, P., Al-Khatib, A., Takrori, F. y Bjertness, E. (2001). Prevalence of Neuropsychiatric and Mucous Membrane Irritation Complaints Among Palestinian Shoe Factory Workers Exposed to Organic Solvents and Plastic Compounds. American Journal of Industrial Medicine, 40(2), 192-198.

Ortiz, A., Izquierdo, H. y Rodríguez, C. (2013). Gestión 
ambiental en PYMES industriales. Interciencia, 38(3), 179-185.

Plaza, J. A., Burgos, J. y Belmonte, L. J. (2011). Grupos de interés, gestión ambiental y resultado empresarial: una propuesta integradora. Cuadernos de Economía y Dirección de la Empresa, 14(3), 151-161. https://doi. org/10.1016/j.cede.2011.02.001

Rodríguez, M., Fernández, Y., Sarmiento, A., Ágreda, O., Bello, M. y Pieters, M. (2014). Ácido hipúrico y efectos neurológicos en trabajadores expuestos a tolueno en una empresa de calzados. Gac Méd Caracas, 122(3), 219-225.

Rodríguez, M. y van Hoof, B. (2010). Para que la PYME sea más competitiva se requiere una gestión ambiental preventiva. https://docplayer.es/7635190-Para-quela-pyme-sea-mas-competitiva-se-requiere-unagestion-ambiental-preventiva-manuel-rodriguezbecerra-bart-van-hoof.html.

Rubio, S., Chamorro, A. y Miranda, F. J. (2007). La investigación sobre gestión medioambiental en la empresa en España (1993-2003). Cuadernos de Economía y Dirección de La Empresa, 10(30), 39-62. https://doi.org/10.1016/S1138-5758(07)70073-7

Vicente-Herrero, M., Capdevila-García, L., TerradillosGarcía, M., Torre, R. I., Victoria, M., Aguilar-Jiménez, E. y López-González, A. A. (2016). Neurotóxicos laborales y contingencia profesional. Comparativa hispano-mexicana. Archivos de Neurociencias, 20(2), 137-147.

Volpentesta, J. R. (2016). Tendencia y perspectiva de la Responsabilidad Social Empresaria. Revista Científica "Visión de Futuro", 20(2), 193-215. 